\title{
La rétroaction corrective à l'écrit et la prise de la conscience linguistique chez les apprenants du français langue étrangère
}

Auteurs : Rania Mohamed Hassan (maître de conférences au département de didactique, université de Damiette, Égypte) et Fatima Zohra Aghlal Boukerma (professeur émérite en sciences de la psychologie, université de Mohamed Bouguerra, Boumerdes, Algérie).

\section{Résumé}

Un bon nombre de chercheurs s'accordent à reconnaître que la rétroaction corrective à l'oral comme à l'écrit peut contribuer à l'acquisition d'une langue étrangère. La rétroaction corrective à l'écrit est une stratégie pédagogique que tout enseignant du FLE doit utiliser, dans le but d'améliorer la conscience linguistique des étudiants. Notre recherche a pour but de vérifier les préférences des apprenants adultes quant à l'utilisation de la rétroaction corrective à l'écrit en classe de FLE en Algérie, de déterminer le type d'erreurs qu'ils commettent le plus souvent dans leurs productions écrites et d'évaluer la pertinence et la mise en œuvre d'une stratégie proposée visant à les aider à mieux comprendre et corriger leurs erreurs écrites. Pour ce faire, 15 étudiants de l'Université M'Hamed Bougara de Boumerdès ont répondu au questionnaire fermé qui leur a été proposé, ont rédigé un texte, ont identifié leurs erreurs soulignées par leur enseignant, ont codé et ont calculé les différents types d'erreurs commises et les ont corrigées. Les résultats ont montré que les étudiants préfèrent l'incitation et la rétroaction métalinguistique plutôt que les autres types de rétroaction corrective. Les résultats ont également indiqué que la stratégie utilisée est efficace. Elle constitue une méthode motivante qui pousse les apprenants à s'autoévaluer et à participer activement au traitement de leurs erreurs. Grâce à cette méthode, une nette amélioration a été observée dans la production écrite des étudiants.

Mots-clés : rétroaction corrective, conscience linguistique, français langue étrangère (FLE), erreurs écrites, auto-évaluation. 


\section{Résumé en arabe}

تساهم التغذية الر اجعة التصحيحية في اكتساب اللغة الأجنبية، فهي بمثابة إستر اتيجية يجب على كل معلم لغة فرنسية أن يلجأ اليها لإثارة الوعي اللغوي لدي طلابه. يهدف البحث الحالي إلي تحديد ما يفضله الطلاب البالغين بالجز ائرمن أساليب لتصحيح أخطائهم الكتابية في اللغة الفرنسية و تقييم مدي فاعلية إستر اتيجية مقترحة لمساعدة الطلاب علي استيعاب وتصحيح أخطائهم بأنفسهم ، وأيضا معرفة انطباعهم عن تللك الإستر اتيجة. تتكون عينة البحث من خمسة عشر طالبا من جامعة محمد أبوكرمة بومدر اس. بعد ان أجاب الطلاب على استبيان مغلق ، قام كلا منهم بكتابة مقال وبعد ذلك قام استاذ المادة بتحديد الأخطاء دون تصحيحها، ثم طلب من كل طالب تصنيف وتحديد نوع و عدد كل خطأ وتصحيحه. كما قام كل طالب بكتابة مقال أخر في نهاية الدراسة. أظهرت النتائج ان الطلاب يفضلون أساليب التغذية الراجعة الغير صريحة خاصة طريقة التحفيز وفيها يشير المعلم فقط للخطأ ليقوم الطالب بتصحيحه بنفسه وطريقة الإثارة اللغوية وفيها يزويدالمعلم الطالب بتعليق أو بمعلومة تساعده على تصحيح الخطأ بنفسه، كما أظهرت الدر اسة مدي فاعلية الإستراتيجية المفترحة في إتارة الوعي اللغوي عند الطلاب وحثهم علي التقييم الذاتي و المشاركة الفعالة في معالجة أخطائهر. 


\section{Introduction}

Plusieurs articles scientifiques montrent que l'erreur commise par l'apprenant dans l'apprentissage d'une langue étrangère (le français, dans notre cas) n'est plus une erreur que l'enseignant doit excuser en la faisant disparaître par la correction, mais bien un indice d'un certain état des connaissances que l'enseignant et l'apprenant doivent s'efforcer d'améliorer, à terme (Marquilló Larruy, 2003). Le terme d'erreur, souligne Cordier (1980 : 13), est réservé aux erreurs systématiques des apprenants. Elles ont trois significations : pour l'enseignant, « elles lui indiquent où en est arrivé l'apprenant par rapport au but visé, et donc ce qui lui reste à apprendre »; pour le chercheur, « elles fournissent des indications sur la façon dont une langue s'apprend, s'acquiert, sur les stratégies et les processus utilisés par l'apprenant dans sa découverte progressive de la langue », et pour l'apprenant, « elles sont indispensables, c'est une façon de vérifier ses hypothèses sur le fonctionnement de la langue qu'il apprend ». Pour Marquillo Larruy (2003: 120), « les erreurs relèvent d'une méconnaissance de la règle de fonctionnement». Par «erreur», nous entendons «toute production d'apprenant qui dévie des modèles de production utilisés par les locuteurs natifs d'une langue » (Calvé, 1992 : 460).

Dans le cadre de l'Algérie, pays à grande majorité arabophone, le français est considéré comme la première langue étrangère et y est enseigné de l'école primaire jusqu'au secondaire. L'arabe et le français présentent d'importantes différences qui renvoient au lexique, à la morphosyntaxe, à la syntaxe de base, à la phonétique, à la prosodie et au graphisme. Ces différences représentent les activités langagières de bas niveau qui portent sur le code linguistique au niveau de la phrase. Les activités de haut niveau, elles, s'attachent à la cohérence textuelle et à l'organisation du discours (Pendax, 1998). Aujourd'hui, ces différentes activités de haut et de bas niveau de la langue française occasionnent de grandes difficultés pour les étudiants universitaires, qui sont orientés vers les langues étrangères, non pas selon leurs vœux ou leurs compétences en FLE, mais selon la moyenne générale au BAC en priorité. Ce sont de futurs enseignants de FLE 
(primaire, collège ou secondaire). Différentes études ont montré que le niveau de la majorité de ces futurs enseignants est assez faible à l'oral comme à l'écrit (Amara, 2009). Dans le contexte européen également, Reinhardt et Rosen (2008) soulignent que les étudiants, même spécialistes de la langue française, futurs enseignants de français, qu'ils soient ou non natifs, n'atteignent pas nécessairement les compétences à l'écrit qui correspondent à la norme académique attendue.

En effet, prêter attention aujourd'hui aux erreurs que commettent les étudiants dans leurs productions écrites et y remédier par la rétroaction corrective en utilisant une stratégie ciblée (d'auto-évaluation) pour l'acquisition du FLE est devenu une nécessité. Selon Facchin (2017), la rétroaction corrective mène à la persévérance et à la réussite scolaire. Par rétroaction corrective, nous entendons la stratégie pédagogique qui est utilisée par l'enseignant dans le but d'améliorer les compétences et les habiletés linguistiques de ses étudiants. Cette amélioration exige évidemment de la part de chaque étudiant qu'il soit attentif et motivé afin de prendre en considération les corrections qui sont faites par l'enseignant et de détecter les règles d'usage sur lesquelles elles portent. Cependant, les étudiants algériens (futurs enseignants de FLE) préfèrent-ils les techniques de la rétroaction corrective qu'adoptent les enseignants lors du cours pour corriger leurs erreurs? Si tel n'est pas le cas, quel est selon eux le type de rétroaction le plus pertinent pour corriger leurs erreurs écrites? Quels sont les types d'erreurs qu'ils commettent le plus dans leur production écrite? Une stratégie d'auto-évaluation ciblée peut-elle avoir un impact sur leurs consciences linguistiques? Comment décrivent-ils les effets de la rétroaction corrective sur leur rapport avec la langue française ? Pour répondre à ces questions, nous nous proposons tout d'abord de présenter le cadre théorique, puis le cadre méthodologique. Enfin, nous analysons les résultats de notre enquête, que nous discutons. 


\section{Cadre théorique}

\subsection{L'approche interactionniste pour l'acquisition du FLE}

Les chercheurs en acquisition des langues semblent aujourd'hui s'entendre sur le fait que l'interaction a une place très importante dans l'apprentissage des langues étrangères. Dans une interaction sont présents deux interlocuteurs (l'enseignant et l'apprenant), qui doivent partager un savoir et un savoir-faire. C'est une situation susceptible de générer de l'apprentissage en poussant l'apprenant à prendre conscience d'une inadéquation entre son répertoire linguistique et ce que présente son enseignant. De ce fait, l'approche interactionniste tend à considérer que les corrections ou les rétroactions correctives, auxquelles est susceptible d'être exposé l'apprenant quand il produit des énoncés en FLE, favorisent le processus d'acquisition dans l'exacte mesure où elles lui permettent de prendre conscience de ces écarts et de les résorber en modifiant les productions non conformes.

En effet, dans l'approche interactionniste, la rétroaction corrective comprend toute correction explicite ou implicite indiquant que la production de l'apprenant n'est pas conforme à la langue cible (Carroll \& Swain, 1993). Autrement dit, la rétroaction corrective est une indication adressée par l'enseignant à l'étudiant visant à lui faire prendre conscience $\mathrm{du}$ fait que son usage de la langue cible n'est pas correct. Une telle caractérisation, selon Marquillo Larruy (2003), repose apparemment sur l'idée d' « erreur ». Aujourd'hui, la notion d'erreur semble abandonnée par les chercheurs en acquisition de langues ; on parle plutôt de productions ou d'hypothèses non conformes à la langue cible. Selon Coşereanu (2009), ces appellations cherchent à traduire l'idée selon laquelle ce qu'on appelle spontanément «erreur» tire son statut d'une référence à un système normatif extrinsèque à l'apprenant (la L2 proprement dite) et qu'il lui faut s'approprier/internaliser, s'il veut hériter de la compétence communicationnelle que l'usage de ce système permet. De son côté, Calvé (1992) considère qu'on ne peut juger que l'étudiant a commis une erreur qu'en fonction de la norme visée, de ce qui a déjà été présenté en classe et du niveau de développement de l'interlangue de l'étudiant. 
L'intrant (les informations auxquelles l'apprenant est exposé) joue un rôle essentiel dans l'acquisition de la langue cible (Gass, 1977). De son côté, Long (1996) souligne le fait que, pour que l'intrant soit compréhensible, il faut qu'il y ait interaction. L'intrant compréhensible facilite l'acquisition, et les modifications langagières effectuées pendant l'interaction favorisent également l'acquisition. Une fonction déterminante de la correction serait donc d'expliciter à l'apprenant les règles et les connaissances sur lesquelles s'appuie sa pratique de la langue, mais dont il n'a pas forcément conscience. La pratique de la rétroaction corrective permet donc de rendre apparente la production non conforme à la langue cible et donnerait ainsi à l'apprenant l'occasion de la réviser. Si l'étudiant est un individu actif qui participe de son plein gré au processus d'apprentissage d'une façon consciente et explicite avec une focalisation, selon Krashen (1982), il est alors possible d'envisager l'acquisition d'une langue en termes de cognition dans une situation interactive où l'apprenant doit réussir la tâche. Pour De Pietro et al., (1989 : 117), il faut insister sur les « séquences orientées vers l'acquisition ». Autrement dit, des séquences qui favoriseraient la prise de conscience (noticing) lors d'une interaction favorisant l'acquisition de la langue cible.

\subsection{La prise de conscience linguistique}

Les différentes productions scientifiques dans le domaine de la rétroaction montrent que la notion de prise de conscience (noticing) dans l'acquisition langagière contribue à notre compréhension de l'apprentissage en interaction. Dans le contexte de l'apprentissage des langues, Nussbaum (1999) évoque la notion de conscience linguistique en incitant les apprenants à prendre conscience de l'écart entre la forme fautive produite (voir les erreurs commises dans leurs productions écrites) et la forme attendue. Ceux-ci sont alors mieux à même de développer leurs compétences langagières. Dans le processus de développement langagier, Ellis (2008) insiste sur l'importance du phénomène de noticing. Selon lui, il s'agit pour l'apprenant de prendre conscience des formes linguistiques auxquelles il est exposé, en particulier quand celles-ci se révèlent non conformes à ses attentes et représentations, en l'état de ses savoirs sur la 
langue. Le même chercheur ajoute que l'enseignant se doit clairement d'attirer l'attention sur la forme : l'éveil à la conscience linguistique par une focalisation sur la forme semble prendre de plus en plus d'essor et contredire la vision d'un enseignement des langues uniquement centré sur les aspects communicatifs, et qui délaisserait ou minimiserait toute focalisation sur le code linguistique.

Lyster (2010: 74) souligne que «les techniques pédagogiques centrées sur la forme attirent l'attention sur des traits langagiers cibles que les apprenants n'utiliseraient pas autrement ou même ne remarqueraient pas dans un contexte d'apprentissage orienté sur la communication ». Cité par Bono (2007 : 25), Ellis (1997) souligne que la prise de conscience (le noticing) est la première des trois opérations que l'apprenant doit effectuer afin de transformer l'input en saisie :

Noticing : l'apprenant focalise son attention sur un point spécifique dans l'input. Comparaison : l'apprenant établit une relation entre ce qu'il vient de noter et l'état actuel de son interlangue.

Intégration : l'apprenant construit une hypothèse de fonctionnement linguistique qui va lui permettre d'incorporer l'élément en question dans son interlangue.

Pour Schmidt (1990), il y a plusieurs niveaux de conscience : la perception, qui n'est pas nécessairement consciente, le noticing, qui demande une attention focalisée, et la compréhension, qui implique l'analyse et la comparaison avec ce qui a été noté préalablement. Parmi les facteurs qui peuvent encourager le noticing, Ellis (1997) distingue la fréquence d'apparition élevée ou encore la saillance d'une forme linguistique, les exigences de la tâche communicative dans laquelle l'apprenant est engagé (en particulier, le besoin de construire le sens de l'interaction avec ses interlocuteurs) et, enfin, les connaissances langagières déjà présentes (perspective développementale). Dans la comparaison, l'apprenant prend conscience de la différence entre les données langagières qu'on lui présente et ce qu'il connaît. Ce traitement des données mènerait, selon Van Patten (1996), à l'intériorisation de l'information (à l'acquisition). 
Pour Van Hest (1996), les apprenants de niveau avancé tendent à focaliser leur attention sur les dimensions discursives des textes produits plutôt que sur les erreurs structurales relevant du bas niveau. Schmidt et Frota (1986) soulignent que, pour que l'acquisition d'une forme linguistique présentée dans l'input puisse avoir lieu, l'apprenant doit porter son attention sur la forme en question et prendre conscience de son fonctionnement. Cela signifie que pour qu'un élément lexical, phonologique, morphologique ou syntaxique de la langue cible puisse être acquis, l'apprenant doit être conscient de son existence. La prise de conscience (le noticing, donc) est une étape nécessaire dans la rétroaction corrective à l'écrit - une rétroaction individualisée où chaque étudiant reçoit des remarques spécifiques sur ses erreurs et ses difficultés linguistiques. En tenant compte de ce qui a été dit au sujet de l'interaction corrective et de la prise de conscience, il semble pertinent de considérer à présent certaines pratiques pédagogiques mises de l'avant par la recherche pour remédier aux erreurs récurrentes.

\subsection{Stratégie et considérations sur la rétroaction corrective}

Harley (2013) explique que les erreurs font partie intégrante de l'apprentissage d'une langue et la tâche consistant à constater pourquoi telle ou telle erreur s'est produite est très importante pour la personne qui cherche à aider l'élève à l'éliminer. Afin d'améliorer la compétence linguistique des étudiants, plusieurs chercheurs (Sheen, 2010; De Gaulmyn, 1992 ; Bisaillon, 1991) semblent d'avis que la rétroaction corrective dans le processus rédactionnel est essentielle pour améliorer la performance en communication écrite. Concernant le rôle des corrections dans le processus rédactionnel, De Gaulrnyn (1992: 199) souligne : «Mieux que le texte achevé et définitif, les brouillons, les ratures, les repentirs, les corrections sont les traces de la gestation du texte et des étapes de sa fabrication. » Pour Bisaillon (1991), la stratégie de correction d'erreurs à l'écrit est une composante importante de la révision du texte ; la correction d'erreurs constitue le processus fondamental de l'activité rédactionnelle. Il incombe aux enseignants d'amener les étudiants à bien utiliser le registre standard du français écrit, en procédant à la correction 
de leurs écrits et en les amenant à s'autocorriger, afin d'améliorer leur niveau à l'écrit. Le rôle de l'enseignant est crucial dans ce processus.

Cependant, nous estimons avec Vigner (2001 : 74) que la plupart de nos étudiants ont encore du mal à corriger leurs erreurs dans des textes écrits. Ils « disposent d'un éventail très limité de modalités de correction. Le texte déjà produit semble d'autre part faire obstacle à un travail de reformulation ». Pour Bisaillon (1991 : 59), les étudiants ne savent pas comment réviser ou s'autocorriger. Ainsi,

Pour qu'il y ait apprentissage de l'écrit, il ne suffit pas d'écrire, les scripteurs doivent comprendre ce qu'est écrire. [...] L'enseignant doit donc expliquer ce qu'implique l'activité rédactionnelle et instruire les scripteurs sur les composantes du processus planification, mise en texte et révision - en mettant l'accent sur le sous-processus de révision puisqu'il est apparu que les bons scripteurs sont ceux qui savent réviser.

Bon nombre de difficultés et d'erreurs en communication écrite auxquelles font face nos étudiants peuvent être résorbées si on leur indique la bonne stratégie. Sheen (2010) précise qu'une rétroaction apportée à l'écrit apporterait de meilleurs résultats qu'une rétroaction donnée à l'oral. En effet, la rétroaction corrective est considérée comme un acte d'interaction entre l'enseignant et l'apprenant. L'interaction permettrait de déclencher ou d'accélérer certains processus développementaux; elle constitue un facteur structurant le processus même de ce développement (Pekarek Doehler, 2000).

Quoi qu'il en soit, afin d'aider les étudiants à prendre conscience de leurs erreurs, le rôle de la rétroaction apparaît primordial. En situation d'interaction, l'enseignant peut attirer l'attention des apprenants sur les aspects formels de la langue (VanPatten, 2004) et par conséquent, l'apprenant peut remarquer la différence entre la forme erronée et la forme correcte fournie par son enseignant (Schmidt, 2001). Cependant, cette pratique doit être répétée et proposée systématiquement si l'on veut qu'elle devienne automatique et efficace. Les étudiants, soulignent Lyster et Mori (2006 : 270), «pourraient avoir besoin d'une rétroaction sur leurs erreurs quand ils ne sont pas capables de découvrir, par la seule exposition à 
l'évidence positive, les points où leur interlangue diffère de la languecible ». Cette revue des concepts et des études relatives à la rétroaction corrective à l'écrit nous ont permis de mieux élaborer nos questions de recherche, que nous énonçons comme suit :

1- Quels types de rétroactions les étudiants (futurs enseignants de FLE) préfèrent-ils pour l'amélioration de leurs compétences linguistiques?

2- Quels sont les types d'erreurs que les étudiants (futurs enseignants de FLE) commettent le plus souvent dans leurs productions écrites ?

3- La stratégie pédagogique ciblée a-t-elle un impact sur la prise de la conscience linguistique des étudiants en FLE?

4- Quelles sont les réflexions des étudiants quant à la l'impact de la stratégie ciblée sur leur rapport avec la langue française?

\section{Méthodologie}

Notre recherche est exploratoire et descriptive et se fixe un quadruple objectif : vérifier auprès des étudiants les types d'erreurs qu'ils commettent; explorer leurs préférences quant aux types de corrections rétroactives fournies; déterminer l'impact de la stratégie pédagogique de rétroactions correctives ciblées sur leurs formes linguistiques erronées à l'écrit, ainsi que déterminer leur réflexion à l'égard de cette stratégie. Nous avons recours à l'approche quantitative, au moyen d'un questionnaire et du relevé du nombre d'erreurs par type, et à l'approche qualitative, comme la stratégie ciblée et son impact.

La recherche a été réalisée dans le cadre d'un cours de neurosciences auquel étaient inscrits les 15 participants ( 8 femmes et 7 hommes). Ce cours fait partie du programme de master(1) (quatrième année universitaire, 2015), spécialité langue française, de la faculté des langues étrangères de l'Université M'Hamed Bougara de Boumerdès en Algérie. Notre échantillon, aux fins de cette étude, se compose de 15 personnes qui ont répondu au questionnaire (première partie de la recherche), tandis que ceux qui ont accepté de participer à l'expérience de stratégie pédagogique (seconde partie de la recherche) sont au nombre de 10. 
Afin de mener à terme cette recherche, nous avons suivi plusieurs étapes avec les étudiants (futurs enseignants du FLE) qui ont pris part à la recherche $(\mathrm{n}=15)$ pour la première partie. Comme outil d'évaluation, nous avons élaboré un questionnaire fermé composé de quatre axes. Le premier concerne les données biographiques ; le deuxième nous permet d'avoir une idée des activités de production écrite effectuées par les étudiants en FLE ; le troisième permet de vérifier l'importance que les participants accordent aux interventions de leurs enseignants à l'écrit; le dernier nous a permis de connaître le type de rétroactions correctives que les étudiants préfèrent. Nous avons soumis le questionnaire aux participants au début de notre expérience.

La seconde partie est très importante quant à la rétroaction corrective à l'écrit. Sheen (2010) souligne qu'une rétroaction apportée à l'écrit génèrerait de meilleurs résultats qu'une rétroaction donnée à l'oral. En effet, nous pensons qu'il y a une rétroaction corrective à l'écrit, d'un côté l'enseignant décide des tâches que les étudiants vont entreprendre et de l'autre, ils recevront par écrit la rétroaction différée. La méthodologie emprunte une approche qualitative qui utilise les données collectées, puisqu'on demande aux étudiants de faire eux-mêmes le codage des erreurs. Quant à l'approche quantitative, elle se présente dans le calcul du nombre d'erreurs produites par les étudiants. Nous souhaitons, par ce travail, évaluer dans quelle mesure la rétroaction corrective en tant que pédagogie ciblée lors d'un cours pourrait pousser les étudiants à être davantage attentifs à la qualité de l'écrit en langue française.

Première étape : De ce fait, la rétroaction à l'écrit permettrait d'éveiller davantage les étudiants à la nécessité de prendre en considération leurs futures productions, ce qui leur permettra de s'autocorriger. Aussi, pour préparer les étudiants et les mettre en confiance pour notre expérience, nous leur avons présenté un texte lacunaire (chose qu'ils ont l'habitude de faire dans le module d'écrit) où ils doivent chercher les erreurs et les souligner.

Deuxième étape : Nous avons demandé aux étudiants du même échantillon qui ont accepté de poursuivre l'expérience $(\mathrm{n}=10)$ de produire 
un texte de vingt lignes (250 mots environ) durant trois heures sur l'importance de la recherche scientifique en FLE, car nous pensons que ce n'est que par la production écrite selon les normes académiques que nous pourrons mettre en évidence les compétences linguistiques des étudiants. Notre objectif principal à cette étape est de mettre en évidence les types d'erreurs que les étudiants (futurs enseignants de FLE) commettent à l'écrit en dépit de leur avancement dans leurs études universitaires (master). Cette vérification d'erreurs nous aidera à envisager la stratégie de la rétroaction corrective.

Troisième étape : Pour recenser les erreurs commises par les étudiants, nous avons récupéré les copies et indiqué les erreurs commises pour chaque étudiant en les soulignant seulement en rouge, sans aucun commentaire ni explication du type d'erreurs.

Quatrième étape : En classe, nous avons remis les copies aux étudiants avec une liste dans laquelle sont identifiés les différents types d'erreurs comme suit :

Tableau 1 : Liste des types d'erreurs

\begin{tabular}{|l|l|l|}
\hline No & Type d'erreurs & \multicolumn{1}{c|}{ Contenu } \\
\hline $\mathbf{1}$ & Orthographiques & $\begin{array}{l}\text { Problèmes avec l'orthographe d'un mot, accents, } \\
\text { ponctuation, majuscules ou minuscules, etc. }\end{array}$ \\
\hline $\mathbf{2}$ & Grammaticales & $\begin{array}{l}\text { Les déterminants (articles : féminin, masculin), } \\
\text { les adjectifs (comparatifs, superlatifs), l'accord } \\
\text { en genre et en nombre, les génitifs et les } \\
\text { composés (noms et adjectifs), la conjugaison des } \\
\text { verbes, les temps, les aspects, les auxiliaires de } \\
\text { modalité, la passivation, autres (gérondifs, } \\
\text { infinitifs) }\end{array}$ \\
\hline $\mathbf{3}$ & Lexicales & $\begin{array}{l}\text { L'utilisation lexicale inexacte, imprécise, } \\
\text { inappropriée ou l'utilisation de mots arabes, } \\
\text { kabyles ou anglais et les mots outils } \\
\text { (articulateurs logiques); les manques dans la } \\
\text { phrase }\end{array}$ \\
\hline
\end{tabular}




\begin{tabular}{|l|l|l|}
\hline $\mathbf{4}$ & Syntaxiques & $\begin{array}{l}\text { L'ordre des mots, les pronoms relatifs, les } \\
\text { conjonctions, les mots de liaison, la ponctuation } \\
\text { et l'orthographe }\end{array}$ \\
\hline $\mathbf{5}$ & Sémantiques & $\begin{array}{l}\text { L'idée de la phrase n'est pas claire, la structure } \\
\text { de la phrase n'est pas correcte. }\end{array}$ \\
\hline
\end{tabular}

Cinquième étape: Nous avons demandé aux étudiants de déterminer les types d'erreurs soulignées dans leurs productions tout en prenant en considération la liste d'erreurs qui leur a été remise. L'objectif, pour nous, est que l'étudiant sache reconnaître le type de chaque erreur commise (qualitatif). Les étudiants ont codifié les données et ont fait un bilan d'erreurs par type.

Sixième étape : Après le codage des erreurs commises, nous avons demandé aux étudiants de calculer le pourcentage d'erreurs pour chaque type (quantitatif). Notre but est de faire sentir à chacun le besoin d'apprentissage en FLE. Pour chaque copie, le codage comme le pourcentage ont été vérifiés par l'enseignant.

Septième étape: Nous avons demandé à chaque étudiant de reprendre sa production, de faire la liste de ses erreurs selon le type dans le but de les corriger en utilisant de la documentation linguistique (en particulier le dictionnaire), de chercher la forme du mot et sa définition, puis d'utiliser un tableau dans lequel on doit trouver : l'erreur, son type et sa correction codés et décodés. Nous avons vérifié l'analyse et la correction des étudiants.

En effet, la rétroaction corrective que nous envisageons dans la présente recherche permet à l'étudiant de prendre conscience de ses difficultés et de ses carences et d'agir avec responsabilité par rapport à son apprentissage en s'autoévaluant et en s'autorégulant. Aussi est-il nécessaire pour les étudiants de réfléchir à leur façon d'apprendre le «réfléchissement» en empruntant la terminologie de Vygotski. Ce réfléchissement est plus précisément lié à l'analyse de contenu faite à partir des productions écrites des étudiants quant à la rétroaction corrective. 
Huitième étape : Nous avons ensuite demandé aux étudiants d'écrire un texte de vingt lignes (250 mots environ) durant trois heures sur l'importance de la recherche scientifique en général en se basant sur les corrections qu'ils ont faites et sur ce qu'ils ont acquis de la rétroaction, et de faire ensuite une comparaison entre la première production et la seconde.

Neuvième étape : À la fin de l'expérience, nous avons demandé aux étudiants d'écrire avec leurs propres termes l'apport de la stratégie pédagogique ciblée quant à l'évolution de leurs compétences linguistiques.

\section{Résultats}

Les résultats du questionnaire ont montré que l'arabe est la langue maternelle de deux tiers des étudiants (10 étudiants) et le kabyle est la langue maternelle d'un seul tiers des étudiants (5 étudiants). Il apparaît que $75 \%$ d'entre eux parlent l'arabe à la maison. Plus de la moitié des étudiants (8 étudiants) parlent trois langues (arabe, kabyle et français) ; 6 étudiants parlent uniquement deux langues et un seul étudiant parle plus de trois langues (arabe, kabyle, français et anglais). À la maison, $80 \%$ des étudiants parlent l'arabe avec les membres de leur famille ou avec leurs amis. Tous les participants (futurs enseignants du FLE) ont commencé à apprendre le français depuis plus de cinq ans, et ils l'ont appris en classe, sauf un étudiant qui a commencé à l'apprendre à la maison. Ces étudiants utiliseront donc le français comme langue d'instruction dans leurs futures pratiques professionnelles.

Concernant la production écrite en FLE, les résultats montrent que $80 \%$ des étudiants aiment de temps en temps écrire en français ; 73,33\% envoient quelquefois des messages écrits à leurs amis dans cette langue ; $20 \%$ d'entre eux le font souvent et $6,67 \%$ n'ont jamais envoyé de messages écrits en français. La plupart des étudiants $(80 \%)$ ont déclaré qu'ils maîtrisent mieux l'écrit que l'oral. Deux tiers des étudiants $(66,67 \%)$ ne sont pas à l'aise quand l'enseignant intervient par rapport à leurs erreurs écrites, tandis que le tiers des étudiants $(33,33 \%)$ se sentent plutôt bien. La majorité des étudiants $(73,33 \%)$ croient que la dictée sous 
toutes ses formes représente l'activité la plus utile pour développer leurs compétences à l'écrit.

Le tableau 2 indique que les étudiants (futurs enseignants de FLE) préfèrent plus d'un type de rétroaction corrective pour l'amélioration de leurs compétences linguistiques. Selon ce tableau, l'incitation et la rétroaction métalinguistique sont les techniques les plus prisées par les étudiants $(26,66 \%)$, tandis que la reformulation et la correction explicite sont les techniques les moins appréciées par eux. Seuls 6,67\% des étudiants préfèrent l'utilisation de ces deux techniques, tandis que 20,01\% préfèrent la demande de clarification. Enfin, 13,33\% des étudiants ont une préférence pour la répétition.

Tableau 2 : Préférences des étudiants quant au type de rétroaction corrective

\begin{tabular}{|l|c|c|}
\hline Type de rétroaction corrective & $\begin{array}{c}\text { Nombre de } \\
\text { réponses }\end{array}$ & $\begin{array}{c}\text { Pourcentage } \\
(\%)\end{array}$ \\
\hline Correction explicite & 1 & $6,67 \%$ \\
\hline Reformulation & 1 & $6,67 \%$ \\
\hline Incitation & 4 & $26,66 \%$ \\
\hline Rétroaction métalinguistique & 4 & $26,66 \%$ \\
\hline Demande de clarification & 3 & $20,01 \%$ \\
\hline Répétition & 2 & $13,33 \%$ \\
\hline
\end{tabular}

En ce qui concerne les types d'erreurs que les étudiants (futurs enseignants de FLE) commettent le plus dans leurs écrits (Question 2), l'analyse de leurs productions écrites nous a permis, dans un premier temps, de mesurer la fréquence et l'évolution des erreurs par type, par 
étudiant et pour le groupe-classe. Le graphique 1 ci-dessous nous permet de visualiser l'évolution des types d'erreurs commises par les 10 étudiants participant à cette activité. Selon ce graphique, les étudiants de master, quels que soient leur cursus en français et les modules de langue qu'ils étudient, commettent des erreurs de langue, en particulier les erreurs orthographiques et grammaticales.

\section{Graphique 1 - Fréquence des types d'erreurs}

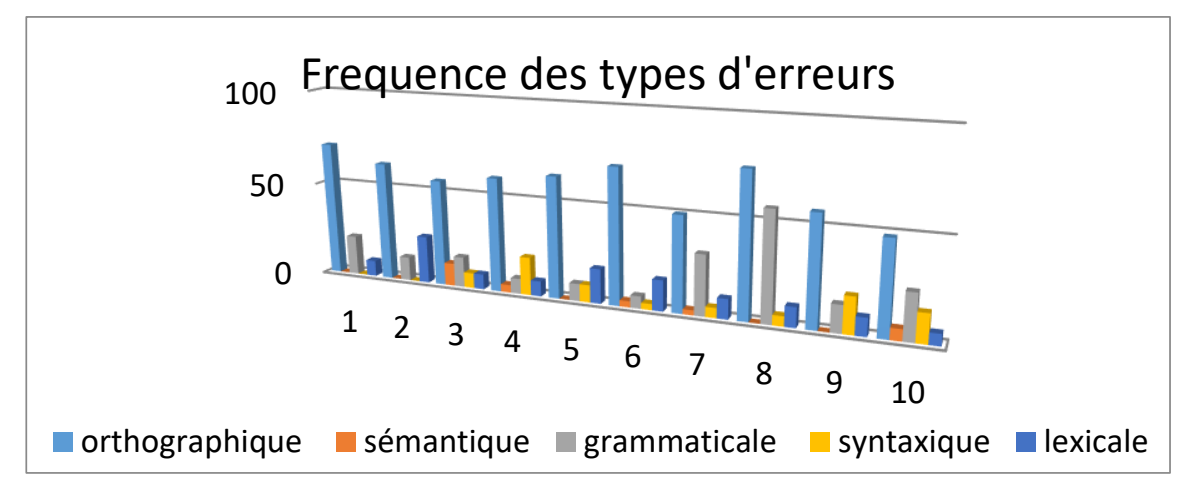

- Le sujet (1) a commis 70,83\% d'erreurs orthographiques, 20,83\% d'erreurs grammaticales et seulement $8,33 \%$ d'erreurs lexicales, mais aucune erreur sémantique ni syntaxique

- Le sujet (2) a commis 62,5\% d'erreurs orthographiques, $25 \%$ d'erreurs lexicales et uniquement $12,5 \%$ d'erreurs grammaticales, mais aucune erreur sémantique ni syntaxique.

- Le sujet (3) a commis $56 \%$ d'erreurs orthographiques, $16 \%$ d'erreurs grammaticales, mais seulement $12 \%$ d'erreurs sémantiques, $8 \%$ d'erreurs syntaxiques et $8 \%$ d'erreurs lexicales.

- Le sujet (4) a commis $60 \%$ d'erreurs orthographiques, $20 \%$ d'erreurs syntaxiques, $8 \%$ d'erreurs grammaticales, $8 \%$ d'erreurs lexicales et seulement $4 \%$ d'erreurs sémantiques.

- Le sujet (5) a commis 63,63\% d'erreurs orthographiques, 18,18\% d'erreurs lexicales, 9,09\% d'erreurs grammaticales, 9,09\% d'erreurs 
syntaxiques ont été commises par le sujet 5, mais aucune erreur sémantique n'a été commise.

- Le sujet (6) a commis 70,96\% d'erreurs orthographiques, 16,12\% d'erreurs lexicales, $6,45 \%$ d'erreurs grammaticales, 3,22\% d'erreurs sémantiques et $3,22 \%$ d'erreurs syntaxiques.

- Le sujet (7) a commis $50 \%$ d'erreurs orthographiques, 31,57 \% d'erreurs grammaticales, 10,52\% d'erreurs lexicales, 5,26\% d'erreurs syntaxiques et 2,63\% d'erreurs sémantiques.

- Le sujet (8) a commis $75 \%$ d'erreurs orthographiques, $15 \%$ d'erreurs syntaxiques, $5 \%$ d'erreurs grammaticales et également $5 \%$ d'erreurs lexicales, mais aucune erreur sémantique.

- Le sujet (9) a commis 57,14\% d'erreurs orthographiques, 19,04\% d'erreurs syntaxiques, 14,28 \% d'erreurs grammaticales, 9,52\% d'erreurs lexicales, mais aucune erreur sémantique.

- Le sujet (10) a commis 48,48\% d'erreurs orthographiques, 24,24\% d'erreurs grammaticales, 15,15\% d'erreurs syntaxiques, 6,06 \% d'erreurs sémantiques et $6,06 \%$ d'erreurs lexicales.

Les résultats montrent que la défaillance sur le plan orthographique (problèmes avec l'orthographe d'un mot, accents, ponctuation, majuscules ou minuscules, etc.) est importante, vu que tous les sujets en commettent $61,45 \%$. Par ailleurs, on observe que les erreurs grammaticales (accord sujet/verbe, participe passé, temps des verbes, etc.) représentent 14,80\% du total des erreurs commises. Les erreurs lexicales représentent 11,47\% ; les erreurs syntaxiques $9,48 \%$, et les erreurs sémantiques $2,79 \%$ du total. Cela correspond respectivement aux moyennes des erreurs commises par tout l'échantillon.

Ces carences langagières, selon nous, peuvent être dues au manque de lecture qui aide les apprenants à enrichir leurs vocabulaires, à améliorer leur style et à utiliser les structures textuelles, à l'influence négative des langues en présence dans le contexte scolaire algérien (en l'occurrence : leur langue maternelle), au manque de rétroaction dans les différents cours disciplinaires et à la non-exploitation de la documentation papier 
(dictionnaires, livres de grammaire, etc.). Cela pourrait aussi être dû au manque d'intérêt des enseignants pour les erreurs langagières commises et au volume horaire assigné insuffisant pour l'apprentissage. En conclusion, nous constatons que le système universitaire, suivant la tradition du système scolaire, accorde très peu d'intérêt à l'évaluation, qui n'est en fait pas un aboutissement, mais un processus. Ce travail met en exergue le rôle de l'enseignant, qui est d'amener l'apprenant à s'autoévaluer et à réfléchir sur son apprentissage.

Pour évaluer l'impact de la stratégie pédagogique ciblée sur la prise de la conscience linguistique des étudiants (question 3), nous avons comparé la moyenne des nombres d'erreurs soulignées par l'enseignant à la première rédaction des étudiants $(16,73)$ et celle à la seconde $(6.53)$. Nous avons trouvé une différence significative $(\mathrm{t}=33.9 ; \mathrm{p}=<0,05)$ entre les deux rédactions en faveur de la première (voir tableau 3 ). C'est-à-dire que le nombre d'erreurs a été diminué à la deuxième rédaction. Cette comparaison affirme l'efficacité de notre stratégie ciblée à éveiller la prise de la conscience linguistique chez les étudiants et à attirer leur attention sur leurs erreurs écrites en FLE.

Tableau 3 : Comparaison entre les deux rédactions

\begin{tabular}{|c|c|c|c|c|}
\hline Rédaction & $\begin{array}{c}\text { Moyenne du } \\
\text { nombre } \\
\text { d'erreurs }\end{array}$ & Écart-type & T & P \\
\hline Rédaction 1 & 16,73 & 2.14 & & \\
\hline Rédaction 2 & 6.53 & 2.16 & 33.89 & 0,001 \\
\hline
\end{tabular}

Quant à la réflexion des étudiants (futurs enseignants de FLE) à l'égard de la stratégie proposée en classe de FLE (question 4), les résultats ont montré que les étudiants apprécient beaucoup la rétroaction corrective, qui leur permet de s'autoévaluer et les incite à revoir les bases et les règles linguistiques de la langue française. Autrement dit, la majorité des 
étudiants affirment apprécier la manière de s'autoévaluer proposée par l'enseignant qui les a incités à être responsables de leur apprentissage, au lieu de leur donner directement la correction.

Dans l'apprentissage de la langue, les étudiants sont appelés à éliminer leurs erreurs par la rétroaction corrective et avec l'aide de l'enseignant. En effet, parmi les apprentissages réalisés par le biais de la rétroaction corrective, les étudiants ont sélectionné les erreurs selon la typologie et ont essayé de les corriger en cherchant la bonne forme et en utilisant différentes documentations (dictionnaires, livres de grammaire, de conjugaison, etc.), méthode qui leur a beaucoup plu et qu'ils souhaitent conserver pour leur apprentissage. C'est ce que l'on observe dans les commentaires des étudiants : «Cette pédagogie nous a incités à utiliser à nouveau de la documentation linguistique pour corriger nos erreurs, chose que je ne faisais pas avant. » La rétroaction corrective a aussi incité les étudiants à revoir certaines règles de grammaire et d'orthographe qu'ils n'avaient pas révisées depuis longtemps, et à faire de même à chaque fois qu'ils doutent d'une certaine forme pour s'en assurer et faire diminuer le nombre d'erreurs dans leurs productions écrites.

Selon les étudiants, en soulignant leurs erreurs écrites sans ajouter de commentaire, l'enseignant a attiré l'attention de l'étudiant sur ses carences et ses difficultés linguistiques. Les étudiants ont également apprécié les orientations de l'enseignant quant à la marche à suivre pour corriger les erreurs : faire la typologie des erreurs soulignées, les compter et les corriger. Ce résultat s'accorde avec l'étude menée par Mc Garrell (2011), qui indique que les étudiants sont plus enclins à se corriger après avoir reçu de rapides consignes portant sur la mécanique de la langue, la grammaire et le vocabulaire que suite à des suggestions de révision portant sur les dimensions discursives-textuelles. Le fait d'établir le pourcentage des erreurs commises est un exercice qui a conscientisé les étudiants quant aux lacunes et difficultés rencontrées dans leur production écrite.

Notons que parmi les 10 étudiants qui ont accepté de faire l'expérience, un étudiant s'est retiré. Pour lui, « ça ne sert à rien de se casser la tête à corriger, puisque l'évaluation dans les modules de spécialité ne 
prend pas les erreurs en considération ». En revanche, la majorité des étudiants étaient très motivés par la rétroaction corrective proposée qui leur permettrait de faire évoluer leurs compétences linguistiques, car ils espèrent continuer leurs études à l'étranger, et cela leur sera très profitable pour préparer leurs tests. Comme le travail avait pour objectif l'apprentissage de la langue française, sans le jugement ni la sanction de la part de l'enseignant, cela a motivé les étudiants à parler entre eux de leurs erreurs et de la manière de les corriger. Cela nous permet de conclure que l'on peut motiver les étudiants à l'apprentissage de la langue française par la rétroaction corrective sans pour autant sanctionner leur production écrite par des remarques ou par une note.

\section{Discussion}

La rétroaction corrective des productions écrites des étudiants s'est avérée être un moyen utile pour faire prendre conscience aux étudiants de leurs carences et de leurs difficultés quant à la maîtrise de la langue française (les erreurs commises) à l'écrit d'un côté, et pour les inciter à s'autoévaluer à l'écrit de l'autre. En effet, la prise de conscience est le point de départ de toute motivation à la correction et à l'apprentissage. L'attention des étudiants a été attirée en premier lieu par le fait que le travail est focalisé sur les erreurs et leur correction selon l'orientation de l'enseignant ; d'autant plus que le rôle de l'enseignant est d'orienter et d'inciter, et non de donner les solutions.

De fait, l'intensité motivationnelle et le désir de l'apprenant à apprendre la langue cible jouent un rôle primordial dans le processus de l'apprentissage (Gardner, 1985). La pédagogie de la rétroaction corrective proposée (le comptage des erreurs commises par chaque étudiant quant à sa production écrite) a permis aux étudiants de prendre conscience de leurs difficultés et de leurs faiblesses linguistiques et a montré l'importance du rôle de l'enseignant dans la rétroaction. Cela a été souligné par Dörnyei (2001), qui insiste sur le fait que l'enseignant doit utiliser des stratégies d'enseignement motivantes. Il doit motiver ses apprenants à accomplir la tâche d'écriture. Selon lui, la rétroaction corrective est un moyen 
permettant de motiver les apprenants à apprendre la langue cible. Elle les aide à prendre conscience de leur progression personnelle.

Dans le même ordre d'idée, les résultats ont montré que le fait de souligner les erreurs commises incite les étudiants à réfléchir au sujet de leurs compétences linguistiques et à la nécessité de corriger leurs erreurs. Dans le même sens, Daigle et Ammar (2013), faisant allusion à l'importance de la correction indirecte, estiment que la correction directe ne permet pas à l'apprenant débutant de mettre en cause sa forme fautive, de réfléchir à son raisonnement ou de remanier ses connaissances. Tandis que dans la correction indirecte, l'enseignant donne un indice à l'apprenant pour qu'il comprenne qu'il y a des erreurs afin qu'il les corrige lui-même. L'enseignant doit donc avoir recours à la correction indirecte. De plus, il doit ultérieurement vérifier la correction de l'apprenant.

La stratégie pédagogique ciblée mise en œuvre pour l'apprentissage de la langue française a mis en évidence les difficultés et les carences des étudiants en matière de grammaire en particulier chez tous les membres de notre échantillon. Nous pensons qu'en rétroaction corrective, la focalisation sur la forme des erreurs dans le but de les corriger, sans recourir à la notation ni à la réflexion au sujet de la non-maîtrise de la langue, a augmenté la motivation à l'apprentissage de l'orthographe, de la grammaire, de la sémantique, etc. en utilisant différentes documentations. Cette étude rejoint les conclusions de l'étude de Ferguson (2011) réalisée auprès de 566 étudiants, qui a montré que les étudiants avaient un avis commun quant à la rétroaction corrective à l'écrit. Ils estiment que la rétroaction corrective aide à augmenter la confiance en soi et que l'encouragement amène à un haut niveau de compétence, tandis que les commentaires trop négatifs de la part de l'enseignant démotivent l'apprenant et peuvent amener les étudiants à abandonner l'apprentissage.

Demander aux étudiants de corriger leurs erreurs en se référant aux documents les a incités à revoir les bases et les règles de la langue cible pour l'autocorrection et les a poussés à améliorer leur acquisition de la langue française. Ce comportement a été encouragé par Guénette (2010), qui a déclaré que les apprenants doivent corriger leurs erreurs eux-mêmes. 
Cependant, la plupart des enseignants de L2 les corrigent eux-mêmes au lieu de guider les apprenants vers l'autocorrection. Pour cela, elle a conseillé aux enseignants d'utiliser les techniques rétroactives incitant les apprenants à s'autocorriger (l'incitation, la demande de clarification, la rétroaction linguistique et la répétition) plutôt que les techniques de rectification (où l'enseignant fournit la forme correcte, comme la reformulation et la correction explicite). L'étude de Bitchener (2008), qui a analysé les effets de la rétroaction corrective à l'écrit sur l'acquisition de l'anglais langue seconde (ALS), a montré que les étudiants qui ont bénéficié de la rétroaction corrective ont réalisé beaucoup plus de progrès que le groupe contrôle.

Par la réécriture du texte, en se basant sur l'autocorrection qui a été faite auparavant, les résultats obtenus ont montré que la correction linguistique des étudiants a été améliorée et que la rétroaction corrective est une pédagogie qui motive les étudiants à faire évoluer leurs compétences linguistiques. Ce résultat converge avec ceux de l'étude exploratoire d'El-Atia et Lemaire (2013), qui ont proposé le portfolio d'erreurs aux étudiants. Les étudiants ont identifié, codifié, corrigé et calculé la fréquence de leurs erreurs. Ils ont également rédigé un texte réflexif. Les résultats obtenus ont montré que la correction linguistique des étudiants a été améliorée et que le portfolio d'erreurs représente un outil pédagogique qui motive les étudiants à participer activement à la correction de leurs erreurs. La rétroaction corrective est donc une stratégie pédagogique que tout enseignant universitaire en langue française devrait utiliser lors de son cours (disciplinaire/linguistique), dans le but d'améliorer les compétences et les habiletés linguistiques de ses étudiants.

\section{Conclusion}

Le but de cette étude exploratoire était d'améliorer la qualité de la langue française écrite des étudiants (futurs enseignants de FLE), en mettant en œuvre la rétroaction corrective comme pédagogie. Un questionnaire fermé a été administré à 15 étudiants de l'Université Boumerdès pour nous permettre de nous faire une idée de la qualité de leurs productions écrites en FLE, d'établir l'importance qu'ils accordent aux 
interventions de leur enseignant et le type de rétroactions correctives qu'ils préfèrent. Le but de cette recherche était également de vérifier si les étudiants seraient prêts à changer leur comportement vis-à-vis de leur apprentissage de la langue cible suite à l'application de la stratégie de rétroaction corrective pour s'autoévaluer en suivant les directives de l'enseignant, sachant que leur travail ne sera pas sanctionné par une note. Les résultats obtenus ont montré que les erreurs orthographiques et grammaticales sont les erreurs les plus commises. Les étudiants sont conscients des différentes techniques de rétroactions correctives. La majorité apprécient davantage les techniques d'autocorrection que les techniques de rectification. Ils préfèrent plus d'un type de rétroaction corrective pour l'amélioration de leurs compétences linguistiques. Ils semblent préférer la correction métalinguistique et l'incitation plutôt que les autres techniques de rétroaction corrective. De plus, la rétroaction corrective a incité les étudiants à revoir certaines règles de grammaire et d'orthographe. Ils ont déclaré qu'ils allaient adopter la stratégie suggérée pour leur futur apprentissage. Les résultats de cette expérience nous encouragent donc à la reproduire avec un plus grand échantillon et à utiliser davantage la rétroaction corrective pour l'enseignement de la langue française au pays.

\section{Bibliographie}

Amara, A. (2001). "Analyse des difficultés rencontrées par des élèves Algériens de 1 ère A. S. dans l'expression des temps verbaux en français ". Insaniyat, $\mathrm{n}^{\circ}$ 15, pp. 189-196.

Bono, M., (2007). "La comparaison L2-L3, un tremplin vers l'acquisition trilingue". Birkbeck Studies in Applied Linguistics, $\mathrm{n}^{\circ}$ 2, pp. 22-41.

Bisaillon, Jocelyne (1991). Les stratégies de révision comme objet d'enseignement, Enjeux, revue de didactique du français, L'Évaluation, 22, 39-54. Namur: CEDOCEF.

Bitchener, J. (2008). "Evidence in support of written corrective feedback". Journal of Second Language Writing, $\mathrm{n}^{\circ} 17$, pp. 102-118.

Calvé, P. (1992). "Corriger ou ne pas corriger là n'est pas la question". The Canadian Modern Language Review/La Revue Canadienne des Langues Vivantes, vol. 48, $\mathrm{n}^{\circ} 3$, pp. 458- 471. 
Carroll, S. et Swain, M. (1993). "Explicit and implicit negative feedback: An empirical study of the learning of linguistic generalization". Studies in Second Language Acquisition, vol. 15, n 3, pp. 357-379.

Cordier P. (1980). "Que signifient les erreurs des apprenants?". Langages, n 57, pp. 917.

Coşereanu, E. (2009). "Le rôle de la correction dans les interactions synchrones entre pairs pour l'apprentissage du français langue étrangère" Cahiers de l'APLIUT, vol. XXVIII, n ${ }^{\circ}$, pp. 39-54.

Daigle, D. et Ammar, A. (2013). "Le codage de la rétroaction écrite : quelques réflexions". Québec français, $\mathrm{n}^{\circ}$ 168, pp. 64-65.

De GAULMYN M.M. (1992). "Rédaction: reformulation et traduction. Lesratures ça nous intéresse...", in R. BOUCHARD (et al.) (dirs.) Acquisition et enseignement / apprentissage des langues étrangères. Grenoble, Université Stendhal, Lidilem.

De Pietro, J.-F., Matthey, M. et PY, B. (1989). Acquisition et contrat didactique : les séquences potentiellement acquisitionnelles dans la conversation exolingue. Actes $d u$ troisième Colloque Régional de Linguistique, Strasbourg 28-29 avril 1988, Université des Sciences Humaines et Université Louis Pasteur, Strasbourg, pp. 99-119. http://mepa.free.fr/STAGE.MEPA.II/planning_fichiers/BIBLIO.Actvite_fichiers/DE \%20PIETRO\%201989.pdf.

Dörnyei, Z. (2001). Motivational Strategies in the Language Classroom. Cambridge: Cambridge University Press.

El-Atia, S. et Lemaire, E. (2013). "Engager les apprenants dans l'auto-évaluation de leurs productions écrites : le cas du portfolio d'erreurs". Revue canadienne de linguistique appliquée, vol. 16, $\mathrm{n}^{\circ} 1$, pp. 111-134.

Ellis, R. (1997). Second Language Acquisition Research and Language Learning. Oxford: Oxford University Press.

Ellis, R. (2008). The study of second language acquisition. Oxford, UK: Oxford University Press.

Facchin, S. (2017). La rétroaction traditionnelle ou technologique? Impact du moyen de diffusion de la rétroaction sur la persévérance et la réussite scolaires (rapport de recherche PAREA no PA-2015-024). Montréal, Québec: Cégep à distance.

Ferguson, P. (2011). "Student perceptions of quality feedback in teacher education". Assessment and Evaluation in Higher Education, vol. 36, $\mathrm{n}^{\circ}$ 1, pp. 51-62.

Gardner, R. C. 1985. Social Psychology and Second Language Learning: The role of Attitudes and motivation. Baltimore, MD: Edward Arnold.

Gass, S.M. (1997). Input, interaction, and the second language learner. Mahwah, NJ: Lawrence Elrbaum Associates.

Guénette, D. (2010). "La rétroaction corrective à l'écrit : Pratiques et croyances, deux réalités parallèles ?" La Revue canadienne des langues vivantes, vol. 66, $\mathrm{n}^{\circ}$ 6, pp. 935966. 
Harley, B (2013). "La langue en jeu dans les classes communicatives de français langue seconde, Ottawa". L'Association canadienne des professeurs de langues secondes, pp.1-107. http://www.caslt.org/resources/french-sl/program-support-intensive-corefrench_en.php

Krashen, S. (1982). Principles and practice in second language acquisition. Oxford, UK: Pergamon Press.

Long, M. H. (1996). "The Role of the Linguistic Environment in Second Language Acquisition". In RithCiE, C. y, BHATIA, T. K. (Éds.), Handbook of Second Language Acquisition. San Diego: Academic Press, pp. 413-468.

Lyster, R. (2010). "Enseignement centré sur la forme et acquisition du genre grammatical en français L2". Revue canadienne de linguistique appliquée, $\mathrm{n}^{\circ} 13$, pp. 73-94.

Lyster, R., \& Mori, H. (2006). "Interactional feedback and instructional counterbalance". Studies in Second Language Acquisition, n ${ }^{\circ} 28$, pp. 321-341.

Marquillo Larruy, M. (2003). L’interprétation de l'erreur. Paris, France: Clé international.

McGarrell, H. M. (2011). ESL students' revisions as a mirror of writing teachercommentary. Communication présentée lors de la conférence annuelle de l'Association canadienne de rédactologie, Université du Nouveau-Brunwick.

Nussbaum, L. (1999). "Émergence de la conscience linguistique en travail de groupe entre apprenants de langue étrangère". Langages, $\mathrm{n}^{\circ}$ 134, pp. 35-50.

Pekarek Doehler, S. (2000). "Approches interactionnistes de l'acquisition des langues étrangères : concepts, recherches, perspectives". Acquisition et Interaction en Langue Étrangère, $\mathrm{n}^{\circ} 12$, pp. 3-26.

Pendanx, M. (1998). Les activités d'apprentissage en classe de langue. Paris, France : Hachette.

Reinhardt, C. et Rosen, E. (2008). "M2 = C2 ? Le CECR, un outil pour penser la place du français dans la construction universitaire européenne". Synergies Europe, n ${ }^{\circ}$, pp. 101-112.

Schmidt, R. W. (1990). "The role of consciousness in second language learning". Applied Linguistics, vol. 11, $\mathrm{n}^{\circ} 12$, pp. 129-158.

Schmidt, R. W. (2001). "Attention". In P. Robinson (Éd.), Cognition and second language acquisition. Cambridge: Cambridge University Press. pp.3-32

Schmidt, R., \& Frota, S. (1986). "Developing basic conversational ability in a second language:A case study of an adult learner of Portuguese". In R. R. Day (Ed.), Talking to learn: Conversation in second language acquisition. Rowley, MA: Newbury House. pp. 237-326.

Sheen, Y. (2010). "Differential effects of oral and written corrective feedback in the ESLclassroom". Studies in Second Language Acquisition, n 32, pp. 203-234. 
Van Hest, E. (1996). Self-repair in L1 and L2 Production. Tilburg, Pays-Bas: Tilburg University Press.

VanPatten, B. (1996). Input processing and grammar instruction: Theory and research. Norwood, NJ: Ablex.

VanPatten, B. (2004). Input processing in second languge acquisition. In B. Van Pattem (Éd.), Processing Instruction: Theory, Research, and Commentary. Mahwah, New Jersey : Lawrence Erlbaum Associates, Inc. pp. 5-31.

Vigner, G. (2001). Enseigner le français comme langue seconde, coll. Didactique des langues étrangères, CLE International, Paris. 